\title{
THE ANNUAL MEETING IN SAN FRANCISCO
}

The seventy-fourth annual meeting of the American Mathematical Society was held at the San Francisco Hilton Hotel in San Francisco, California, from Tuesday through Friday, January 23-26, 1968. There were 3,826 persons in attendance, including 2,743 members of the Society. This meeting of the Society was held in conjunction with the annual meeting of the Mathematical Association of America.

Professor Eugene P. Wigner of Princeton University delivered the forty-first Josiah Willard Gibbs Lecture, entitled Symmetry principles in old and new physics. Professor Wigner was introduced by Professor C. B. Morrey, Jr.

Dean A. A. Albert of the University of Chicago delivered the Presidential Address, the title of which was On associative division algebras. He was introduced by Professor C. B. Morrey, Jr.

By invitation of the Committee to Select Hour Speakers for Annual and Summer Meetings, two invited addresses were given at the meeting. Professor Wolfgang Wasow of the University of Wisconsin spoke on Connection problems for asymptotic series solutions of ordinary differential equations. Professor H. A. Antosiewicz presided at this session. Professor Louis Auslander of the City University of New York presented $A$ survey of solvable Lie groups and applications. He was introduced by Professor Reinhold Baer.

Professor Jurgen K. Moser of the Courant Institute of Mathematical Sciences, New York University was awarded the first George David Birkhoff Prize in Applied Mathematics. The award was presented by Professor S. M. Ulam. Professor J. Barkley Rosser presided at this session.

There were two special sessions of twenty-minute papers. The topic of the first session was Integration in function spaces. It was organized by Professor Monroe Donsker of the Courant Institute of Mathematical Sciences, New York University, and consisted of talks by Professor R. H. Cameron, University of Minnesota; Professor Leonard Gross, Cornell University; Professor Edward Nelson, Princeton University; Professor Mark Kac, Rockefeller University; Professor S. R. S. Varadhan, New York University; and Professor I. E. Segal, Massachusetts Institute of Technology. The second special session of twenty-minute papers was devoted to Piecewise linear topology. Professor Morris W. Hirsch of the University of California, Berkeley, arranged the program which consisted of papers by Professor Marshall Cohen of Princeton University and Professor Dennis Sullivan of the University of California, Berkeley; Professor E. H. Connell, University of California, Berkeley; Professor T. F. Banchoff, Brown 
University; and Dr. C. P. Rourke of the Institute for Advanced Study, Princeton, and the University of Warwick, and Dr. B. J. Sanderson of the University of California, Berkeley and the University of Warwick.

There were thirty-five sessions for contributed papers, at which the following persons presided: Professor L. W. Anderson, Professor Carlos Borges, Professor C. E. Burgess, Professor W. J. Coles, Professor R. A. Duke, Professor Bernard Epstein, Professor J. M. G. Fell, Professor S. W. Golomb, Professor R. B. Guenther, Professor C. J. A. Halberg, Jr., Professor A. W. Hales, Professor C. A. Hayes, Professor R. W. Heath, Dr. T. R. Jenkins, Professor R. A. Knoebel, Professor Shoshichi Kobayashi, Professor W. M. Krueger, Dr. R. B. Leipnik, Professor Joaquin Loustaunau, Professor J. L. McGregor, Professor Henryk Minc, Professor L. W. Neustadt, Professor R. J. Nunke, Professor K. L. Phillips, Professor Edmund Pinney, Professor P. T. Rygg, Professor H. J. Ryser, Professor G. T. Sallee, Professor A. K. Steiner, Professor Takayuki Tamura, Professor H. G. Tucker, Professor F. A. Valentine, Professor B. J. Walsh, Professor J. H. Walter, Professor T. W. Wilcox, and Professor D. E. Wulbert.

The Council met on January 22, 1968 at 2:00 P.M. in the Walnut Suite of the San Francisco Hilton Hotel.

The Council was informed that the Executive Committee approved a meeting on November 8-9, 1968 at Clemson University and a Symposium on Mathematical Questions in Biology jointly with Section A of AAAS in December 1968.

The following persons represented the Society at the events respectively noted:

John J. Bowers at the inauguration of Paul Francis Geren as President of Stetson University

Cathleen Morawetz at the inauguration of Martha E. Peterson as President of Barnard College

Elliott L. Buell at the inauguration of Frederick Herbert Jackson as President of Clark University

Ralph P. Agnew at the seventy-fifth anniversary of the founding of Ithaca College

John H. Hodges at the inauguration of Maurice B. Mitchell as President of the University of Denver

Robert B. Sloan at the inauguration of the Very Reverend Reginald A. Redlon, O.F.M., as President of St. Bonaventure University

John A. Ferling at the inauguration of Robert Christian Kramer as President of California State Polytechnic College

Walter J. Klimczak at the inauguration of Archibald M. Woodruff as Chancellor of the University of Hartford 
The following committee appointments by President Morrey were entered in the record:

Committee to Nominate Candidates for Membership on the Executive Committee: A. A. Albert, N. E. Steenrod, Daniel Zelinsky.

Nominating Committee for 1968 Election: A. P. Calderon, E. R. Kolchin (chairman), G. D. Mostow, R. S. Palais, A. H. Taub.

Replacements on Applied Mathematics Committee: J. B. Diaz, H. S. Wilf (extending term). This is a joint committee with SIAM. President Birkhoff of SIAM appointed Hirsch Cohen and Richard Varga. Continuing members are Jim Douglas, Jr., and W. H. Reid.

Replacements on Committee on Translations: J. C. Moore, R. S. Phillips. This is a joint committee with The Institute of Mathematical Statistics. President Harris of IMS has appointed J. I. Rosenblatt. Continuing members are H. A. Antosiewicz, F. Browder, V. Klee, I. Olkin (representing IMS).

Committee to Select Gibbs Lecturers for 1969 and 1970: K. O. Friedrichs, M. Kac, D. H. Lehmer.

Tellers for 1967 Election: C. W. Saalfrank, J. P. Crawford.

Replacements on Committee on Summer Institutes: Louis Nirenberg, E. H. Spanier.

Replacement on Committee to Select Hour Speakers for Summer and Annual Meetings: D. C. Spencer.

Replacement on Committee to Select Hour Speakers for Eastern Sectional Meetings: Fritz John.

Replacement on Committee to Select Hour Speakers for Southeastern Sectional Meetings: A. W. Goodman.

Replacement on Committee to Select Hour Speakers for Western Sectional Meetings: Louis de Branges.

Replacement on Committee to Select Hour Speakers for Far Western Sectional Meetings: Errett Bishop.

Added to Organizing Committee for a Symposium on Numerical Solutions of Field Problems in Continuum Physics: Jim Douglas, Jr., S. Fernbach, R. S. Varga, C. H. Wilcox.

Organizing Committee for a Symposium in Applications of Categorical Algebra: Hyman Bass, Alex Heller (chairman), J. C. Moore.

Organizing Committee for a Symposium on Combinatorics: Marshall Hall, T. S. Motzkin (chairman), G.-C. Rota.

Organizing Committee for a Symposium on Non-Linear Functional Analysis: F. Browder (chairman), James Eells, T. Kato, G. J. Minty, R. S. Palais, J. T. Schwartz, S. Smale. 
Committee on Modification of Time and Effort Reporting: G. W. Mackey (chairman), R. S. Palais, Alex Rosenberg.

The Council received the reports of Executive Committee business by mail that the following 574 nominees of institutional members were elected to membership:

University of Alabama: Jeannette M. Smith.

University of Alberta: Eric D. J. Buckley, Mrs. Chinthayamma, Myron Goldberg, Chandra Mohan Pareek, Jagdish Prasad, George E. J. Smith,

Andrewes University: Carlyle D. Flemming, Burckhard J. Glass, Clifford O. Pope. Antioch College: Howard W. McCay III, Roger N. Metz.

University of Arizona: Jacob A. Brandler, Douglas J. Hoylman, Mrityunjay S. Samanta.

University of British Columbia: Walter Allegretto, Andrew B. Carson, John J. Crawford, Michael J. Friedlaender, Anthony T. Lau, Hugh G. R. Millington, Douglas W. Maclean, Ole A. Nielsen, S. David Promislow, Hilal A. Qureshi, Patrick N. Stewart, Alexander C. Walker, James C. Wong.

Brooklyn College: Philip F. Ancona, Michael J. Dalezman.

Brooklyn Polytechnic Institute: Howard Anton, Denis L. Blackmore.

Brown University: Kin-Etsu Abe, Allan Howe, Kenneth J. Preskenis, Patrick J. Ryan, Toshio Takahashi.

Bucknell University: James O. Stevenson.

California State College at Fullerton: Peter N. Hoefsmit, Clyde J. Labate, James E. Vandezande.

California State College at Long Beach: Rocky L. Nelson, Gary J. Sexton.

University of California, Davis: Paul F. DuBois, Grant W. Labarre, Martin K. McCrea, Mark B. Sadler, Curtis C. Travis.

University of California, Los Angeles: William L. Armacost, Gerald P. Farrell, Carl E. Gordon, Keith S. Joseph, Edward Y. Mikami, Louis E. Narens, Richard A. Tapia, Peter G. Tripodes, Alan J. Truelove.

Carnegie-Mellon University: Pushpa P. Agashe, Utpal K. Bandyopadhyay, George Y. Chi, William A. Day, Linnea D. Imler, Martin A. Leonardo, Robert L. Rosenberg, Maurice D. Weir.

University of Chicago: David L. Golber, David M. Goldschmidt, Charles P. Lanski, Robert Sandling, Julius L. Shaneson, Richard W. Sullivan, Richard B. Tarsy, Paul R. Wolfson.

City College: Alan Landman, Ora E. Percus.

Connecticut College: Walter F. Brady.

Cornell University: Woodrow D. Brownawell, I. Lok Chang, George F. Feissner, Victor W. Goodman, Harvey J. Iglarsh, Robert D. Little, Manju Mather, Robert A. Morris, Kalyan K. Mukherjea, Brian K. O'Byrne, Jacob M. Plotkin, Robert D. Speiser, Ellen R. Stone, Hajime Taniguchi, John F. Yeagley.

Dalhousie University: Ena M. Roberts.

Dartmouth College: Arunas Rudvalis, James L. Williams.

Duke University: Glenn A. Bookhout, William C. Dunning, Roger C. Grimson, Barry L. Lobb, James B. Lucke, E. Ohmer Milton, Humphrey H. Nash, Joseph C. Nichols, Jeffrey L. Piech, James C. Reber, Carl G. Wagner.

East Carolina University: John B. Davis Jr, Frank W. Saunders, William R، Spickerman.

Emory University: David M. Clark, Caulton L. Irwin, Glenn F. Webb.

Florida Presbyterian College: Terry L. Hartsook, Robert C. Meacham Jr.

Florida State University: John M. Baker, Phillip E. Brown, John T. Hofler, Brian R. 
O'Callaghan, Stephen W. Smith, John R. Wilkerson.

Franklin and Marshall College: Robert T. Glassey, George L. Greenwood, Randall M. King.

Georgia Institute of Technology: James J. Buckley.

University of Georgia: Genevieve E. Gaither, Hartmut Hoft, Kreso Horvatic, George J. Michaelides, Jurgen Schmidt, Philip W. Walker, Winston W. Walker.

Harvard University: Richard A. Berthold, Michael Crampin, Robert J. Fisher, Alan

S. Friedman, David Gieseker, Gerald W. McCollum, Seppo U. Rickman, Steven

D. Shnider, Richard P. Stanley, Peter D. Taylor, Jonathan M. Wahl.

University of Houston: Frank J. Hall, Jerry V. McBee.

Humboldt State College: Henry S. Tropp.

Idaho State University: John W. Grahl, Michael P. Johnston, Raymond G. Lewin. University of Idaho: Robert I. Matthews.

Illinois Institute of Technology: Martin G. Buntinas, Philip Calabresse, Ping-Wen Chao.

University of Illinois: Henry Louis Africk, William J. Barlow, Mary E. Beattie, Edward D. Bender, John G, Bergman, Michael M. Brady, John W. Chaney, Burgess J. Davis, Catherine A. Doleans, Joe W. Fisher, Patrick J. Fleury, Lowell J. Hansen, Steven N. Iverson, Harold Jacobs, David H. Johnson, H. Randall Johnson, Jerry A. Johnson, Gudrun Kalmbach, Robert L. Long, David L. Madison, Tapas Mazumdar, Robert R. McConnell, Dean P. McCullough, Allan W. McInnes, John K. Moore, Grace G. Orzech, Oliver R. L. Pretzel, Balram S. Rajput, Kenneth B. Reid, Jr., Hla H. Sein, Kenneth R. Slonneger, Arthur R. Solomon, Ahmed R. Sourour, Steven Takiff, Shukichi Tanno, Riho Terras, Ann E. White, Chi Song Wong, William L. Young.

Indiana University: James A. Deddens, Jonathan K. Lee, Ramesh M. Kulkarni, P. Ramankutty, William C. Ridge, James W. Roberts, Louis E. Smogor Jr., Warren R. Wogen.

Institute for Advanced Study: Jonathan P. Brezin, Andreas W. M. Dress, Herve M. Jacquet, Kiyosato Okamoto, Hideki Omori, Shunichi Tanaka, Claude A. Weber, James E. West.

Ithaca College: Dianne D. Schmidt, Carleton R. Worth.

John Jay College: Alexander Joseph.

Johns Hopkins University: Gerald A. Backman, John B. Baillieul, Abje Feintuch, Joyce E. Maples, Frederick W. Meier Jr., Toshitsune Miyake, John E. Vernaglia.

University of Kansas: Joseph A. Bergin, Ronald E. Cote, Felix F. Dreher Jr., Jerald P. Dauer, Thomas F. Ewing, Joseph A. Gallian, John D. Harris, David E. Kullman, Deborah E. Prince, Robert A. Sulanke, Carl E. Taylor.

Knox College: Peter R. Politi.

Lehigh University: Siham C. Braidi, James Guyker, George A. Huff, Peter G. Jessup, Edwin J. Kay, Stephen B. Leonard, Steven J. Michaels, George O. Schneller IV, Nathan F. Simms Jr., Stanley L. Stephens, Louis W. Stern, John W. Taylor. LeMoyne College: Diana J. Marotta, James W. Uebelacker.

University of Maine: Stephen E. Godsoe, Stephen M. Perry, Franklin H. Shelton Jr. Marquette University: Richard J. O'Farrell, Faustine L. Perham, Joan N. Quenan. University of Maryland: Mary Lou Buttram, Shui-Nee Chow, Craig M. Cordes, Richard S. Davis, Robert A. Morris, Robert I. Winston, Gordon S. Woodward, Chin S. Yu.

McGill University: Antonio W. De Souza, Robert Benjamin, Harry Greenspan, Derek A. Holton, Harold W. Proppe, Robert M. Raphael, Jonathan J. Shuster. McMaster University: Frank Bozel, Joseph W. Lorimer, Gary Spoar.

University of Miami: William G. Bany, Shih-Yung Chung, Richard Corbin, Ralph A. 
Fernandez, Hector I. Hirigoyen, Jung-Shan Lin, Osvaldo Marrero, Gary L. Musser, David Rudd.

University of Michigan: Donald P. Ballou, Richard F. Baum, John C. Breckenridge, David H. Culver, Alexander K. Dewdney, Joseph G. Ecker, Allan R. Hoffer, Kenneth P. Johnson, Edward L. Keller, Herbert C. Lyon, Bennet Manvel, Richard W. Marshall, John R. Mertens Jr, James J. Metzger, Richard C. Misare, Kenneth R. Rebman, John H. Remmers, Walter Tape, Alan H. Shuchat, Richard F. E. Strube, Mavina K. Vamanamurthy, Michael Zeiger.

Mississippi State University: Charles H. Nicholas.

University of Missouri, Columbia: Elwyn H. Davis, William A. Ettling, Ronald L. Sinzdak.

University of Missouri, St. Louis: Michael D. Scroggins.

University of Nebraska: Lynn H. Erbe, Gene A. Klaasen, Richard L. Tangeman.

University of New Hampshire: Kay L. Clauson, Stephen A. Kenton, Marshall J. Kotzen.

University of North Dakota: Thomas P. Wiggen.

Ohio State University: Henry W. Block, Frederick S. Koehl, Clifford S. Queen.

University of Oklahoma: James A. Anderson, Lilia D. Chance, Joe W. Knickmeyer, George C. Morrow.

University of Oregon: Ronald P. Brown, Theodore C. Burrowes, Wei-Ching Chang, Stephen A. Book, David S. Browder, Edward N. Ferguson, Ronald D. Grisell, Ray O. Hamel, Douglas N. Hawley, Peter J. Horn, Bruce R. Johnson, Michael R. W. Kervin, William F. Lipman, Norman C. Meyer Jr., Andrew L. Perrie, John R. Spooner Jr., Michael B Tomlinson, Milton E. Wannier.

University of Ottawa: Mike P. Closs, G. K. Radhakrishna Rao, Sin L. Tan

Pennsylvania State University: Robert T. Burger, Frank A. Chimenti, David J. Devries, John C. Feduska Jr., Robert J. Fornaro, Robert E. Hall, Grace PingPoo Ho, Richard M. Jones, Charles F. Kelemen, Roger H. Marty, John R. Metzner Jr., Edward A. Parberry, Wilbur L. Smith, Jung H. Tsai, William R. Weller, David E. Zitarelli.

Princeton University: Thomas E. Armstrong, Stephen S. Gelbart, Frank E. Gerth III, Thomas S. Hammerstrom, Soren A. Illman, Terry G. Lyons, Paul D. Magriel, Lewis Shilane, Neal W. Stoltzfus, Mark L. Yoseloff.

Purdue University: Joseph L. Bertorelli, Michael Brand, Ben-Ami Braun, Kevin F. Clancey, Raymond F. Dembek, Gwilym Edmunds, Robert J. Ellison, Euline I. Green, John P. Helm, Gerald R. Hintz, William C. Jones, Jongsik Kim, Richard B. Lane, Ta-Fend Lin, Charles D. Luning, Richard F. Melka, Guillermo Miranda, William Nelson, Steven A. Schonefeld, Tsong-Chih Shieh, James E. Shirey, Harris S. Shultz, George Szeto, John F. Weidner.

Rensselaer Polytechnic Institute: Stanley Kogelman, Timothy A. Loughlin, David J. Wollkind.

Rice University: Joseph A. Becker, Roger Keith Dennis, Brian P. Gerber.

University of Rhode Island: Constantin Corduneanu, Anilchandra A. Kayande, M. Rama Mohana Rao.

University of Rochester: Richard M. Aron, William M. Bush, Ronald C. Freiwald, Arun V. Jategaonkar, Dennis F. Kibler, Nand Lal, Ellen Orfinger, Walter Read, Eleanor L. Rockwell.

Rutgers, The State University: Ralph A. Artino, Aronon M. Bedner, Richard A. Decarli, Robert W. Earles, Jerrold Fischer, Patrick M. Fitzpatrick, Northrup Fowler III, Richard A. Guhl, David P. Kopcso, Ann L. Koski, Jeffrey M. Levine, Reginald K, Luke, Daniel A. Marcus, Marie H. McKellar, Richard E. Munson, Mary Jane Simpson, Charles Unger, Ira K. Wolf.

St. Olaf College: John A. Feroe, Pamela A. Myhr, Richard A. Peik. 
San Diego State College: John H. Griffin, Lawrence R. Weill, Brian D. Wick. University of Saskatchewan: Rolland J. Gaudet, Kee Seng Ong, Chin Yong Teh, Ting On To.

University of Saskatchewan, Regina: Kenneth K. Chao, Stuart A. Rankin, James Thomas Smith.

Simon Fraser University: Richard E. J. Fraser, Soo-Seng Siah.

South Dakota School of Mines and Technology: Judy F. Doering, Daniel E. Lipkie, William Torrie.

Southern Colorado State College: Thomas J. Bartlett.

Southern Methodist University: Jacqueline S. Hall, Dovalee D. McElroy, Raymond C. Sansing.

Stanford University: Yehoshafat S. Giveon.

Stephen F. A ustin State College: Jack D. Chapman, Joseph E. Roos, Susan J. Schrey. Stevens Institute of Technology: Jeffrey M. Brown, Robert W. Quackenbush, David B. Preston.

Syracuse University: Douglass L. Grant, Neil L. Millman, Brian B. Moore, Joan M. Murphy, Walter J. Pugh, William H. Reynolds, Herb Silverman, John R. Walker, Andrew R. Wohlgemuth.

University of Tennessee: Edwin C. Anderson, William S. Boyd, Stanley R. Chadick, William H. Dent Jr., John G. Fryman, Eleanor R. Jones, Laura M. Lawson, Michael W. Mislove, Calvin I. Owens, Donald C. Ramsey, Charles R. Wall. Texas $A$ and $M$ University: Alexander F. Kleiner Jr., Steve Ligh.

Texas Technological College: Jonathan S. Burton, Sterling G. Crossley, Dennis A. Johnston.

Trinity College: Lucy D. Deephouse, Lucien A. Gehami.

University of Toronto: James B. Whitney.

Vanderbilt University: Lee H. Minor.

Virginia Polytechnic Institute: Robert M. Porterfield, Frederick R. Ward, James D. Wine.

University of Washington: Hector A. Barrios, David S. Birkes, John E. Coury, Robert P. Kurshan, Robert B. Moore, Pullamplam K. R. Nathan, Clark E. Runnion, Michael Schulzer, George B. Williams, Joseph Zaks.

Washington University: Walter A. Carrington Jr., Mei Chen, Mark J. Driscoll, Nikolaas J. H. Heideman, J. Myron Hood, Glenn A. Lancaster Jr, Norman E. Liden, Robert B. Putz, Gillian G. Raw, Floyd L. Williams, Edward N. Wilson.

Wayne State University: Khalid Benabdallah, Gary S. Ellenson, Robert B. Hardin Jr., Joseph D. Hrycyseyn, Sanford Perlman.

Wellesley College: Mary R. Hoover, David M. Pritzker.

Wells College: Ray C. Shiflett.

Wesleyan University: Neil B. Hindman, Chong-Min Koh, Sidney R. Tims, ZongHwe Tzeng.

Western Michigan University: Daryl M. Brink, Jerome J. Karaganis, John A. Mitchem, Douglas W. Nance, Kenneth L. Pothoven, Shrikant Narayan Rao, Gerald L. Sievers.

Western Washington State University: Larry A. Curnutt.

College of William and Mary: John J. Avioli, Joyce F. Longman, Turner L. Robinette $\mathrm{Jr}$.

University of Wisconsin: Christina A. Bahl, Michael P. Benson, Vasily C. Cateforis, Alfred S. Cavaretta Jr., Graham A. Chambers Jr., Edward W. Chillak, Charles W. Congo, Richard C, Detmer, George W. Dinolt, Fred W. Dorr, Edgar E. Escultura, Charles E. Hughes, Alvin R. Jaffin, George S. Jordan, Paul D. Kindschi, Richard A. Kramer, Anthony W. Leung, Jonathan W. Lewin, Dar-Biau Liu, Dean A. Neumann, Jitendra N. Manocha, Carol S. Murphy, Dennis M. 
Parra, Steven D. Purisch, John Read, Arthur Richards Jr., Edward B. Scrimger Jr., Charles S. Sinnett, Dean E. Smith, Jerald H. Tutsch, Peter R. Wadsack, Robert L. Wheeler, Joella Yoder, Albert L. Zobrist.

University of Wisconsin-Milwaukee: Madhukar G. Deshpande, Harvey S. Fox, Gerald L. White.

Yale University: Mark Benard, John W. Brookes, D. Bruce Erickson, Miguel A. Garcia-Negron, Michael G. Henle, Karl E. Petersen, Leonard F. Richardson, James P. Ryan, Jon H. Sanders.

The Secretary announced the election of the following one hundred sixty-one persons to ordinary membership in the Society:

Adkins, Kirby T. II, St. John's University;

Allard, Sister M. Raimonda, Rosary College;

Altman, Yale, Blaisdell Publishing Company, Waltham, Massachusetts;

Ancheta, Caesar R., University of Texas at El Paso;

Arenberg, Carne, Argonne National Laboratory, Argonne, Illinois;

Atherton, Charles R. Jr., Washington State University;

Balintfy, Joseph L., Tulane University;

Baylock, Adrian F., St. Francis College;

Becker, David N., Washington University;

Blais, Herve, Externat Classique de Longueuil, Jacques-Cartier, Quebec, Canada;

Bolder, Arthur R., Brooklyn College;

Bonnett, Dennis Alan, Northern Arizona University;

Bourke, Donald R., Groton Public Schools, Groton, Massachusetts;

Bowman, Deanna G., Appalachian State University;

Brumfiel, Gregory W., Princeton University;

Buchert, Edwin W., Wisconsin State University, Whitewater;

Burke, Barbara L., New York Stock Exchange, New York, New York:

Burke, David Paul, Catholic University of America;

Burniston, Ernest E., North Carolina State University at Raleigh;

Burrell, Benjamin A., Ohio State University;

Canty, John T., University of Notre Dame;

Carlson, John A., Whitworth College;

Chen, Albert T., U. S. Geological Survey, Menlo Park, California;

Cohen, Paul I., Yale University

Colton, David L., Indiana University;

Crocker, Roger Clement, Queen Elizabeth College, London, England;

Crockett, Robert N., University of Washington;

Datta, Subhendu K., Indian Institute of Technology, Kanpur, India;

Davis, Ronald M., University of Maryland;

Dickey, Ronald Wayne, University of Wisconsin;

Dreyer, Thomas P., Stellenbosch University, Stellenbosch, Africa;

Easton, Robert W., Brown University;

Eden, Allison R., London College of Applied Science, London, England;

Edmonstone, George R., University of Notre Dame;

Ellis, Christopher F., Data Dynamics Corporation, Ft. Walton Beach, Florida;

Erdelyi, Ivan N., Kansas State University;

Erlbaum, John F., Jersey City State College;

Farlow, Stanley J., National Institute of Health, Bethesda, Maryland;

Faulkner, John R., Yale University;

Fearnley-Sander, Desmond P., California State College at San Bernardino;

Ferguson, John D., Institute for Defense Analyses, Princeton, New Jersey;

Findley, David F., University of Frankfort, Frankfort, Germany;

Fincke, Nancy C., Western Illinois University; 
Fortin, Jacques G., Laval University, Quebec, Que., Canada;

Fox, Stewart B., Jr., U. S. Army, Ft. Belvoir, Virginia;

Fredricksen, Harold M., California Institute of Technology;

Fried, Martin, Pratt Institute;

Gable, David Lee, Washington and Lee University;

Gopal, Mangalam R., Michigan Technological University;

Goswick, Thomas E., U. S. Naval Weapons Laboratory, Dahlgren, Virginia;

Grant, James L., Urban Engineers, Atlanta, Georgia;

Grasser, Horst-Siegfried P., University of South Africa, Pretoria, Africa;

Graves, William H., University of North Carolina, Chapel Hill;

Guignard, Monique M., University of Lille, Lille, France;

Guitart, Miguel A., Alabama College;

Gunderson, Robert W., National Aeronautical and Space Administration, Huntsville, Alabama;

Gustafson, Richard F., State University of New York, College at Oneonta;

Handlan, James M., Susquehanna University;

Hansen, Rodney T., Montana State University;

Hanwell, David C., U. S. Air Force, Norton Air Force Base, California;

Hedetniemi, Stephen T., University of Iowa;

Heinrich, Raymond Lee, DeVry Institute of Technology;

Heller, Dorothy M., 20102 Chateau Drive, Saratoga, California;

Higgins, Stanley B., Texas Christian University;

Hilliker, David Lee, University of California-Irvine;

Hollister, Herbert A., Bowling Green State University;

Hosey, Ronald R., Boeing Aircraft Corporation, New Orleans, Louisiana;

Howard, Fredric T., Wake Forest University;

Hudson, William N., University of California-Irvine;

Jones, Neil D., Pennsylvania State University;

Khazanie, Ramakant G., University of Vermont;

Kiesler, Franz, Bellarmine College;

Kim, Young Jip, Taejon Presbyterian College, Taejon, Korea;

Kiung, Sidney H., Jacksonville University;

Knowles, Joan B., U. S. Bureau of Public Roads, Washington, D. C.;

Kohlmeyer, Richard J., Hartwick College;

Kreutzer, Howard P. Jr., P and M Finishing Company, Long Island City, New York;

Leake, Charles R., Wagner College;

Levien, Edward, 1520 W. 22nd Street, Miami Beach, Florida;

Lientz, B. Price, University of Washington;

Loc, Nguyen X., University of Aarhus, Aarhus, Denmark;

Linden, Theodore A., Fordham University;

Lindsey, Linda M., Tarleton State College;

Liu, Ming-Tsan, University of Pennsylvania;

Lo, Chi Yeung, Michigan State University;

Lomen, David O., University of Arizona;

Lord, Graham F., State University of New York at Buffalo;

Macarow, Leo, Chicago School for Adults, Chicago, Illinois;

Mahrous, Mohamed-Amina, Wichita State University;

Mamelak, Joseph S., Community College;

Marzocco, Mauro J., Fairleigh Dickinson University;

Mathisas, Adrian R. D., Trinity College, Cambridge, England;

Max, Nelson Lee, University of California-Berkeley;

Mezzino, Michael J., University of Texas;

Miller, Robert L., Sandia Corporation, Albuquerque, New Mexico;

Miller, Shirley G., Montgomery County Community College; 
Minton, Hubert L. Jr., Memphis State University;

Moore, J. Douglas, State University of New York, College at Oswego;

Most, Mel O., Structure Language and Number Research Project, Ridgefield Park, New Jersey;

Mowshowitz, Abbe, University of Michigan;

Mukherjee, Some N., Banaras Hindu University, Varanasi, India;

Mulholland, Robert J., Washington University;

Neath, Walter J., Northern Illinois University;

Newell, David C., Tufts University;

$\mathrm{Ng}$, Kung-Fu, University of Swansea, Swansea, Wales;

Niemann, Ralph H., Colorado State University;

Nunley, Clyde Dale, Southwestern at Memphis;

Ow, Wellington H., California State College at Fullerton;

Papadopoulos, Peter G., DeVry Technical Institute;

Paschke, William L., University of Oregon;

Pazy, Amnon J., Stanford University;

Peters, Helen M., State College at Westfield;

Phillips, Robert G., University of South Carolina;

Plaumann, Peter K., University of Michigan;

Plivka, Andrew D., New York Institute of Technology;

Pounder, James R., University of Alberta;

Pausson, Harvey R., University of Kentucky;

Priestley, William M., University of the South;

Pringle, Eugene C., State University of New York, College at Oneonta;

Rahimi, Morteza A., University of Iowa;

Ramos, Gerardo, Institute of Mathematics, Lima, Peru;

Reid, Wilson G., General Electric Company, King of Prussia, Pennsylvania;

Rivera, Dorothy M., San Diego State College;

Robertson, Harold G., Murray State University;

Russo, John P., Andrews University;

Sabater, Manuel P., Bicol Development Planning Board, Legazpi City, Philippines;

Sacker, Robert J., University of Southern California;

Saint Mary, Donald F., Iowa State University;

Saylor, Paul E., University of Illinois;

Schneider, Robert B., Cornell University;

Seitelman, Leon Harold, 50 Hillside St., East Hartford, Connecticut;

Senechal, Marjorie, Smith College;

Shershin, Anthony C., University of South Florida;

Sigrist, Francois, University of British Columbia;

Silverman, Mitchell L., Brooklyn College;

Simons, William H., Carnegie-Mellon University;

Singer, Ivan, Institute of Mathematics, Bucharest, Rumania;

Skwarczynski, Maciej, University of Warsaw, Warsaw, Poland;

Snygg, John, Upsala College;

Starkey, Joel B., Aero Chart and Information Center, St. Louis, Missouri;

Stoian, Eliodor R., National Energy Board, Ottawa, Ont., Canada;

Strange, William E., Mississippi College;

Sykora, Ronald G., U. S. Naval Radiological Defense Laboratory, San Francisco, California;

Tavathia, Bikram S., University of Manitoba;

Taylor, Charles D., Addison-Wesley Publishing Company, Reading, Massachusetts;

Thornber, Edgar H., University of Chicago;

Thurston, Rachel F., University of California, Berkeley;

Thurston, William P., University of California, Berkeley; 
Toczycki, Wladyslaw A., Institute of Gas Technology, Chicago, Illinois;

Trott, William R., U. S. Air Force, Arlington, Virginia;

Valenti, Nicholas W., Hauppauge School District, Hauppauge, New York;

Varma, Arun Kumar, University of Alberta;

Verdonk, Thomas H., Northwestern Mutual Life Insurance, Milwaukee, Wisconsin;

Wade, Luther I., Louisiana State University;

Walters, J. Allen, U. S. Naval Radiological Defense Laboratory, San Francisco, California;

Williams, S. Irene, Educational Testing Service, Princeton, New Jersey;

Wilson, Robert Lee, Yale University;

Wood, Bruce, University of Arizona;

Wood, James Alan, Georgetown University;

Woodward, Richard M., Ford Motor Corporation, Dearborn, Michigan;

Younger, Daniel H., General Electric Company, Schenectady, New York;

It was announced that the following had been admitted to the Society in accordance with reciprocity agreements with various mathematical organizations:

Australian Mathematical Society: David S. Asche, Erich W. Ellers, Joachim A. Hempel, Michael P. O'Donnell.

Österreichische Mathematische Gesellschaft: Gerd Baron.

Dansk Matematisk Forening: Henrik Stetkaer-Hansen.

Societe Mathematique de France: Claude P. Bruter, Maurice Chacron, Roland A. Guy.

Deutsche Mathematiker Vereinigung: Albrecht H. Abele, Horst E. H. Becker, Wolfgang Hackenbroch, Siegfried Thomeier.

The London Mathematical Society: Frank Robert Drake, Felix A. E. Pirani, Hans P. Rogosinski.

Indian Mathematical Society: Prem Chandra Consul, Udita Narayana Singh.

Israel Mathematical Union: Abraham Berman, Jacqueline B. Shalhevet, Lior Tzafriri, Ezra Zeheb.

Mathematical Society of Japan: Tutomu Iio, Koichi Iwata, Kazuo Kishimoto, Tetsuo Kodama, Shoji Kyuno, Teishiro Saito, Masamichi Takesaki.

Real Sociedad Mathematica Espanola: Miguel L. LaPlaza.

Schweizerische Mathematische Gesellschaft: Franz Streit.

Also, that the following institutions were elected to institutional membership:

University of Dallas, Dallas, Texas

Western Georgia College, Carrollton, Georgia

University of Wyoming, Laramie, Wyoming

East Carolina University, Greenville, North Carolina

University of Calgary, Alberta, Canada

Angelo State College, San Angelo, Texas

Northern Illinois University, DeKalb, Illinois

State University College, Oswego, New York

Pace College, New York, New York

Muskingum, College, New Concord, Ohio

Morehouse College, Atlanta, Georgia

Also, that the First National City Bank, New York, New York was elected to Corporate Membership.

A meeting at The Johns Hopkins University on October 26, 1968 was approved. 
The Council approved a recommendation of the Executive Committee that the Society publish cover-to-cover English translations of the journals Matematičeskir Sbornik (New Series) and Izvestija Matematičeskaja Serija. In each instance the translation is to begin in 1968 with the numbers appearing in the original in 1967.

The Council approved the replacement during January 1968 of W. H. J. Fuchs as member of the Proceedings Editorial Committee by Maurice Heins.

The Council noted that Professors Jacob Burlak of Duke University and Chester Feldman of Kent State University will become Associate Editors of Mathematical Reviews during the summer of 1968 and that at about the same time Dr. Richard J. Crittenden, now Associate Editor, will become Executive Editor upon completion of the term of Dr. S. K. Berberian.

The Council approved procedures for continuing future production of indexes for Mathematical Reviews. It will result in a five year index through 1969 to appear in 1970.

The Council approved the selection of Professor Harish-Chandra as a Colloquium Lecturer in 1969.

Following its recess for dinner, the Council considered a set of problems raised by the large number of contributed ten minute papers. At the current meeting, where a limit of 350 papers had been set on the number of papers to be scheduled, the number contributed was nearly 650 . The Council voted to retain ten minute papers at Society meetings without limitation on the number. [The Secretary wishes to make several observations. First is the fact that the Council was well aware of the problems of time and space associated with scheduling a large number of contributed papers. Second is the consensus, not embodied in a formal resolution, that there should be ample time at each meeting for hour addresses and symposia. Third is the inevitability of an earlier deadline for the submission of abstracts of contributed papers for the Annual Meeting.]

The Council adjourned at 8:50 P.M.

The Business Meeting was convened at 11:15 A.M. by Associate Secretary R. S. Pierce in the absence of the President. The Secretary called the attention of the membership to the open meeting of the Committee to Monitor Problems in Communication scheduled for the following evening. He reported various actions of Council on the previous day. The Business Meeting adjourned at 11:30 A.M.

Bethlehem, Pennsylvania

Las Cruces, New Mexico
Everett Pitcher

Secretary

Richard S. Pierce

Associate Secretary 